\title{
Medical Image Fusion Using Unified Wavelet Principal Component Technique
}

\author{
Tran Duc Tan \\ Associate Professor in Electronics Engineering, Faculty of Electrical and Electronic Engineering, \\ Phenikaa University, Hanoi, Vietnam \\ tranduc@phenikaa-uni.edu.vn
}

Received: $24^{\text {th }}$ February 2021, Accepted: $19^{\text {th }}$ March 2021, Published: $30^{\text {th }}$ April 2021

\begin{abstract}
Medical imaging is a vital diagnostic and therapeutic tool. For the patient's treatment, high-quality pictures are needed to correctly outline the target volumes and structures. For diagnosis, treatment planning, and patient followup in the management of patients with a blood tumor, imaging data are utilized measurement and calculation of therapeutic dosages are necessary for treatment planning. For treatment planning, MRI and CT scans alone do not provide enough geometric and physical information. The benefits to the patient from merging these photos show the importance of image fusion in medical imaging. Doctors can utilize the simulation results in this paper to diagnose and analyze images from a variety of modalities with ease thanks to the use of MATLAB software.
\end{abstract}

Key Words: Medical Images fusion, MATLAB, Inverse Wavelet Transform

\section{Introduction}

Images captured with a multispectral camera often reveal details that aren't visible to the naked eye. Infrared, ultraviolet, X-ray, auditory, and radar data are all examples of this. This isn't an image in the traditional sense because the information it represents cannot be seen by the human eye directly. Thematic Mapper and Multi Spectral Scanner pictures, for example, map the various spectral bands to RGB components to portray the data visually.

When two or more images from various imaging modalities are combined into a single image that retains significant information from each, the process of image fusion is called.

Image fusion is a popular and important method to interpret image data for a more suitable image for a variety of applications such as visual interpretation and digital classification, Aerial and satellite imaging, Robot vision, concealed weapon detection, Multi-focus image fusion, digital camera application, battle field mapping,

The following characteristics characterize an effective picture fusion technique. Because it preserves so much relevant information from many photos, it's a great tool. First and foremost, it does not produce artefacts that could confuse or deceive a human observer or any later image processing stages. Third, it must be dependable and durable. Finally, it should not remove any relevant data from any of the photos that it receives.

Data fusion is a method of combining data and information from several sources to produce more accurate information for making decisions. "The merging of two or more separate images to create a new image by employing a specific algorithm" is the most common definition of image fusion. The goal of image fusion is to combine data from multiple sensors to provide a more comprehensive picture than would be possible with only the data from a single sensor. An image F is created by fusing two separate images A and B and transferring their data into a single new image $F$.

It is possible to gain additional clinical information when images from several modalities are combined. As a result, radiologists prefer utilizing a variety of imaging modalities in order to gain a more comprehensive picture. All the useful information from each modality is extracted and integrated into a single image using image fusion. For the most part, the result of a successful picture fusion should be devoid of any artefacts or inconsistencies. 


\section{Literature review}

An Effective Wavelet-Based Approach to Medical Image Fusion was presented by Yong Yang et al. in 2010. ' The pixel averaging, inclination pyramid, discrete Wavelet Transform, and a viable wavelet-based methodology are studied for two different datasets here. Strictly following the procedures that have showed promising results in our initial experiments, we've chosen which sets of data to use. Standard Deviation, Average Intensity, Information Entropy, and Cross Entropy are used in the statistical analysis of data. All of the factors have been compared by the writers, making it simple to decipher what they have found.

Two-scale picture fusion with saliency detection was proposed by Bavirisetti and Dhulli. Saliency extraction was carried out using this technique, which can bring to light important details. When compared to the multi-scale fusion technique, this effort produced superior results. The medical photographs were not processed correctly using this procedure. Wavelet-based picture fusion on FPGA was presented by Pemmaraju et al for the first time. Xilinx EDK 10.1 and Spartan 3E were used to achieve this new approach. For high-speed applications, this FPGA has combinational blocks. Memory, flip-flops, and a look-up table (LUT) are all part of this design. This technology was used to combine images taken by different cameras. There are no stationary outputs from the DWT, and the low frequency component is less efficient.

It was proposed by Yang and coworkers that multi-model fusion of images might be achieved using fuzzy logic. In order to get low and high bands, NSCT was examined using a pre-registered source picture. Local energy algorithm employs low-frequency bands. All sub-bands of inverse NSCT were used to create the proposed fused image. Additionally, the accuracy, contrast, and variety of the data were assessed. Low spatial resolution is the fundamental downside of this technique. When Shivsubramani Krishnamoorthy and colleagues discussed the fusion algorithms in 2010, they introduced the development of combination procedures, different fusion calculations and the linking of intertwined images for picture informational index and further determined picture quality measurements like mean square error, PSNR, average distinction, structural substance, normalised cross correlations, maximum contrast, and normalised absolute error for breaking the link.

These settings have been credited with enhancing image clarity and definition. For digital camera picture combination calculation in 2015 N.sravani, et al. presented their work on the IHS model and PCA based combination as well as multiple scale change based combination like Brovey, High pass sifting and diverse pyramid approaches like Gaussian, Laplacian or Gradient as well as morphological and Wavelet based as well. We've finally come up with the metrics RMSE and PSNR to measure the exhibition An MATLAB Graphical User Interface (GUI) model for therapeutic picture combination and analysis of multiple wavelets using different parameters was provided in 2016 by researchers Rajesh Bhandar and colleagues as Wavelet-based Analysis of Medical Image Fusion using MATLAB GUI.

\section{Research Methodology}

Wavelet theory: Wavelets are a relatively new development in the realm of signal processing, yet they provide a versatile method for decomposing signals at several resolutions. This method, known as the wavelet transform, allows you to break a function down into its constituent frequency components, allowing you to examine each one at a resolution that corresponds to its size. It may thus be claimed that the wavelet transform is a time-frequency localization tool, which means that an event is linked to the time it occurred. Zweig's discovery of the continuous wavelet transform in 1975 (formerly known as the cochlear transform and discovered while examining the response of the ear to sound) has made significant contributions to wavelet theory. Since then, multi-resolution analysis and orthogonal wavelets have been developed. The Fourier Transform (FT) has previously been the most often used. Non-stationary signals, such as those with time-varying spectra, cannot be analysed using FT, which has a major downside. A true time-frequency representation of a signal requires a transform that is time and frequency 
dependent because most biological signals are non-stationary. Using the wavelet transform, we can represent time and frequency.

Principal component analysis (PCA): When a data matrix of $\mathrm{n}$ objects is broken down into linear combinations of $\mathrm{p}$ variables, Principal Component Analysis (PCA) can be used to summarise the data using uncorrelated axes (principal components). Variation among items is shown in the first $\mathrm{k}$ components. Each of the $\mathrm{p}$ variables is represented as an $\mathrm{n}$-point cloud in a multidimensional space. The mean of each variable is used to get the centroid of the locations.

An algorithm known as principal component analysis (PCA) is used to transform a large number of correlated variables into an uncorrelated set of principle components. An efficient and succinct description of the data collection is generated by this algorithm. Most of the data variation is accounted by the first principal component, and the remaining variance is accounted by each subsequent component. The direction with the greatest variation is assumed to be the first main component. To ensure that the second primary component does not interfere with the first, it must be positioned perpendicular to it. Component points in the direction of maximum variance in this particular subspace. A subspace perpendicular to the first two is used to extract the third and subsequent principal components, with the direction of maximum variance as the starting point. The Karhunen-Loève transform, or the Hoteling transform, is another name for the PCA. The basis vectors for PCA, unlike those for FFT, DCT, and wavelet transforms, are determined by the set of data used.

Unified wavelet principal component (UWPC): It is a typical way to reduce the dimensions of hyperspectral images whose projections have the propensity to have the highest variance conceivable for $\mathrm{N}$-spectral subspace at all values of N. PC (Principle component), is a data adaptive ortho normal transform. Spatial information is not taken into account by the PC. The spectral image is made up of a large number of unlabeled pixels.

Edges and picture data can be easily represented on a variety of spatial scales using wavelets. Improving filters is as simple as applying a scale factor to the wavelet coefficients on the image. For many tasks, wavelets outperform pixels. As a result, in order to optimise the amount of edge information in images with smaller pixel dimensions, we explore reducing the Wavelet coefficient's PC dimension. A wavelet transforms each image band, but a PC transforms all of them at once. This means that the two changes can be used in distinct contexts. The pixels' Eigen spectra will be the same for PC and the complete set of wavelet coefficients.

However, PC can be applied on a subset of wavelet coefficients in order to find Eigen spectra that optimise the energy of this subset. Because of this, Eigen spectra generated by the PC will only maximise vertical wavelet energy in certain subbands. It is called "integrated wavelet PC" because it uses a masked or modified wavelet coefficient set to identify the Wavelet PC Eigen spectrum, and then projects the picture to the Wavelet PC Eigen spectrum. As a result, images of reduced dimension can be enhanced without artefacts being filtered out by the computed projection basis.

To compute the PC wavelet, the PC wavelet is based on the own spectra of the scaled wavelet (e.g masked) and a transformation of modified wavelet. When the pixel image is projected onto the own spectrum, and the main component photographs are computed with wavelet pictures, and then transforming the inverse wavelet, the fused image is obtained after performing canny edge detection on fused image for better diagnosis. 


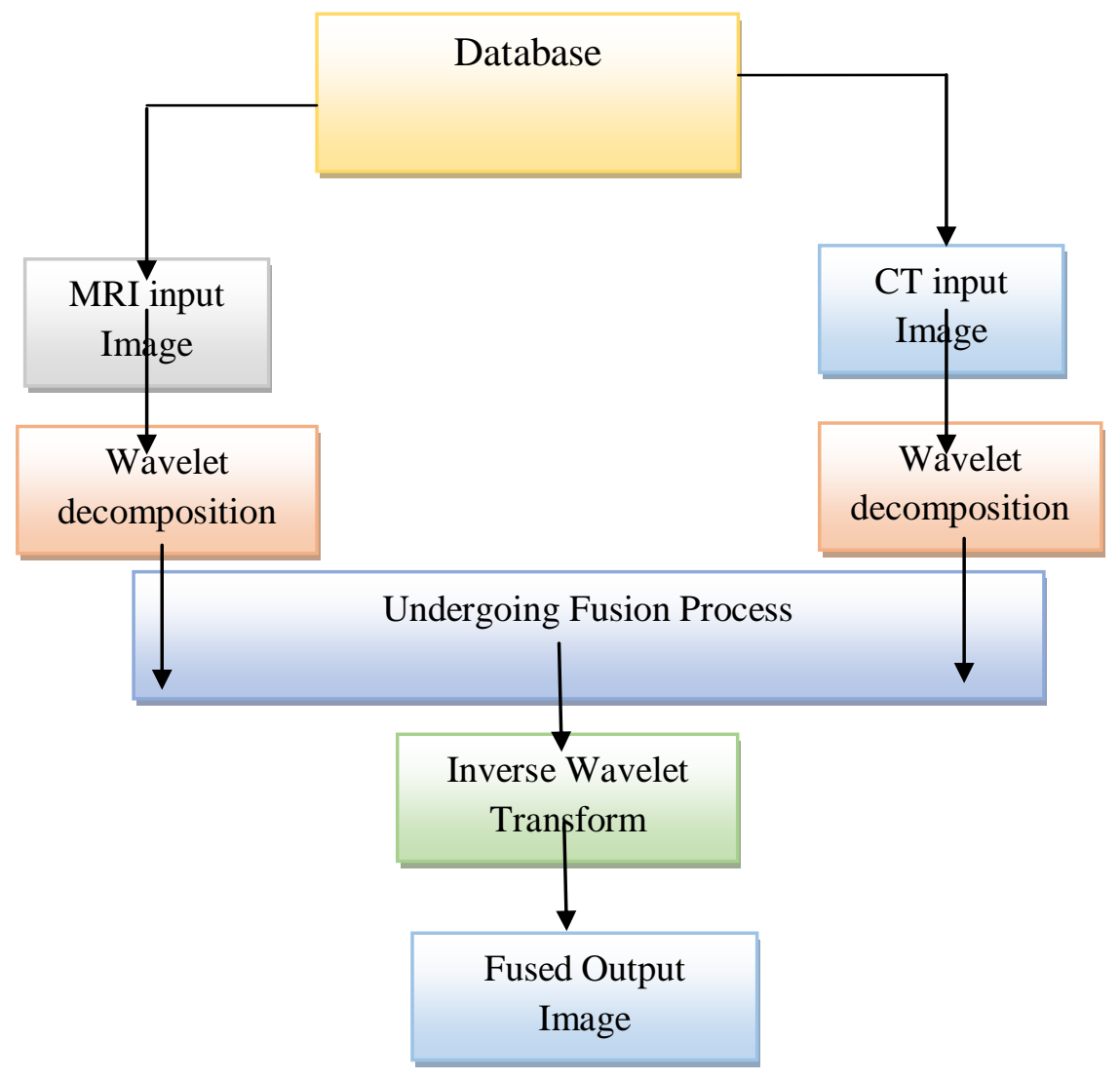

Fig 1: Block Diagram of Unified Wavelet Principle Component

\section{Results and Analysis}

The images in the figures below depict the input and output images of several fusion processes. Figures $\mathrm{a}$ and $\mathrm{b}$ are the input images from the MRI and CT image databases, and figures $\mathrm{c}$ through $\mathrm{r}$ are the output images.

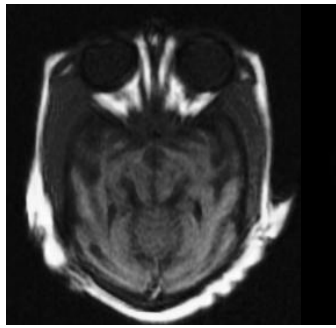

(a)

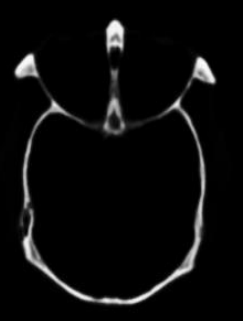

(b)

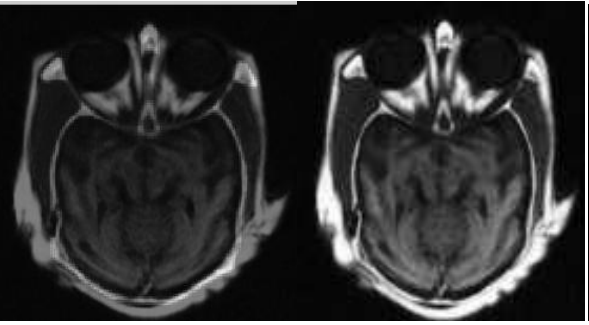

(c)

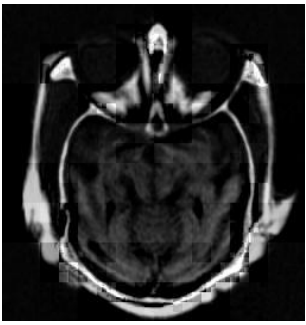

(e)
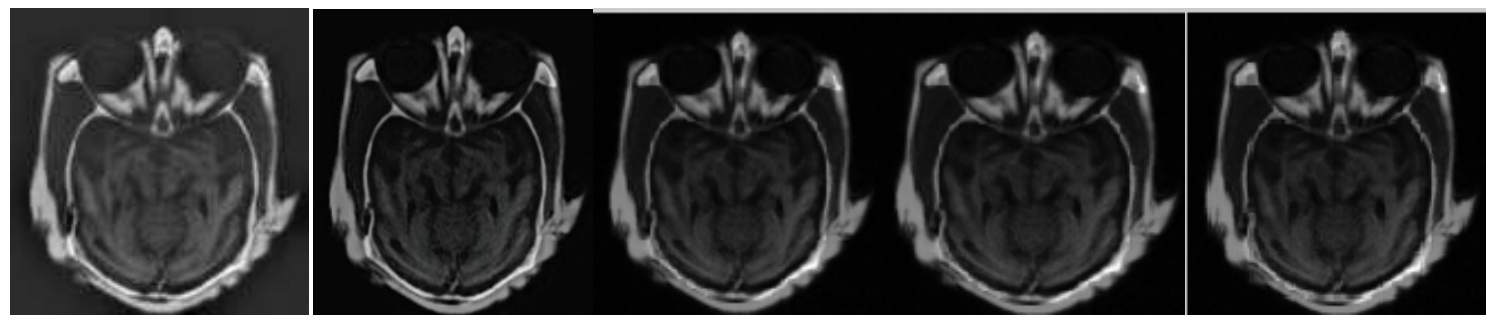
(f)

(g)

(h)

(i)

(j)

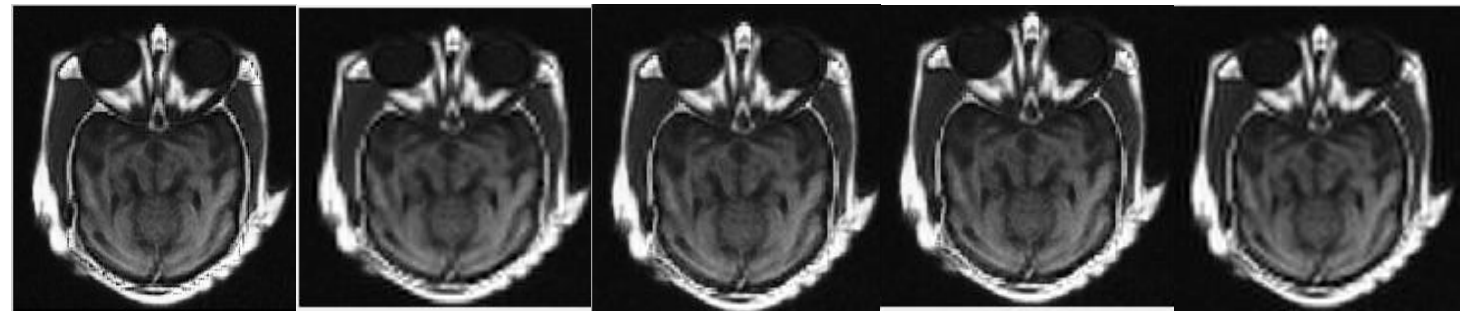

(k)

(1)

(m)

(n)

(o)

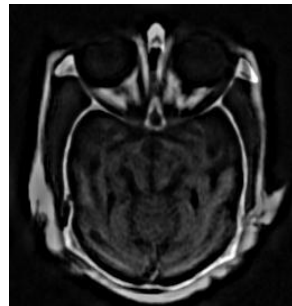

(p)

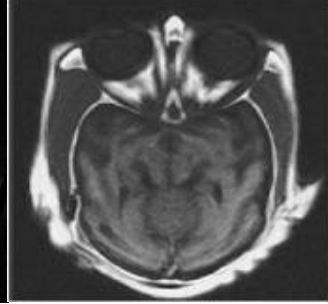

(q)

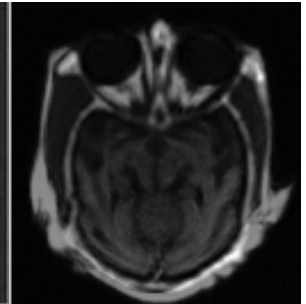

(r)

Figure 2: (a) MRI image (b) CT image (c) Average fusion (d) PCA (e) DWT

(f) Laplace (g) gradient (h) orthogonal (i) Biorthogonal (j) Trous (k) Haar (l) Daubechies (m) Coiflets (n) Symlets (o) Meyer (p) DTCWT (q) RWT (r) IWPC

The below figures represent the canny edge images of fused images of various fusion techniques

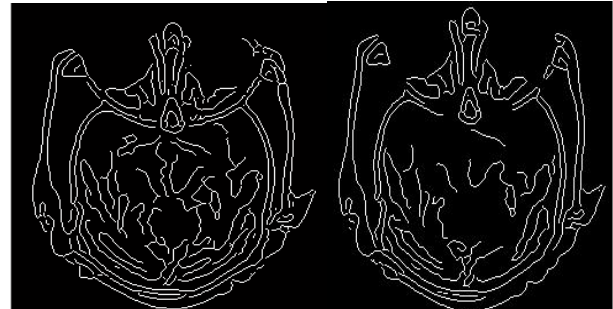

(a)

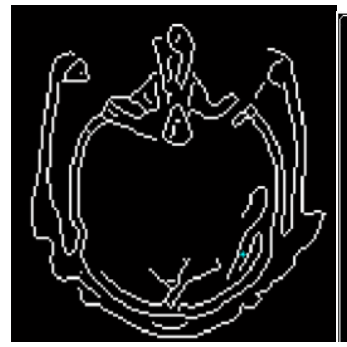

(f) (b)

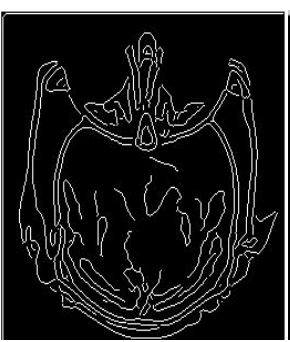

(g)

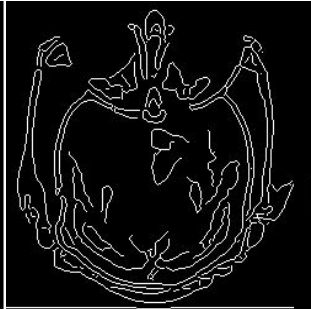

(c)

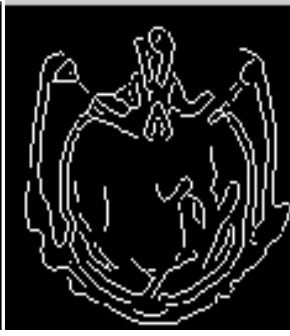

(h)

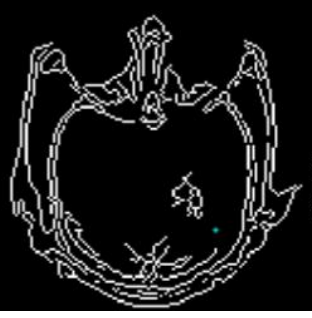

(d)

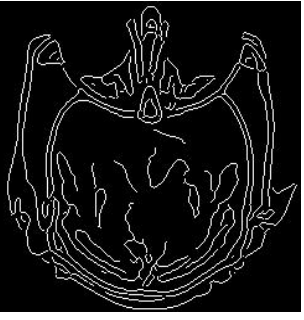

(e) 


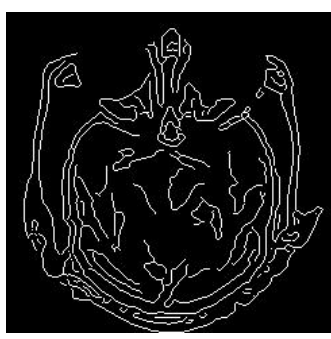

$(\mathrm{k})$

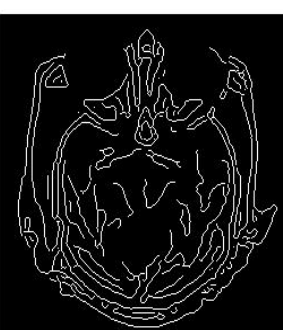

(1)

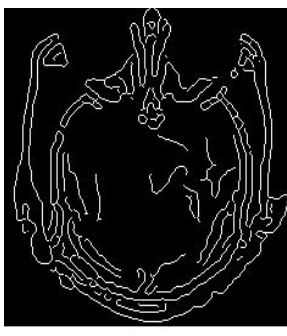

(m)

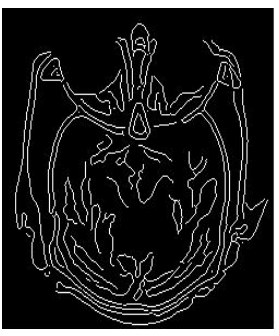

(n)

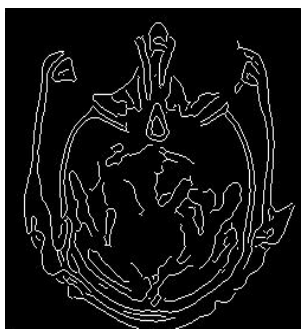

(o)

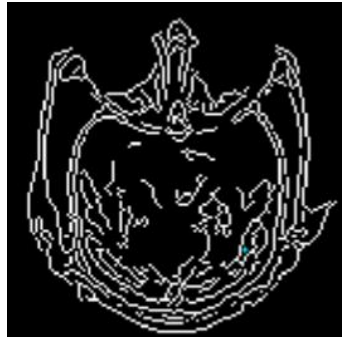

(p)

Fig 3: (a) Average fusion (b) PCA (c) DWT (d) Laplace (e) gradient (f) orthogonal (g) Biorthogonal (h) Trous (i) Haar (j) Daubechies(k)Coiflets(l)Symlets(m)Meyer (n) DTCWT (o) RWT (p) IWPC

For the purpose of analysing the output visually as well as statistically using quality measuring parameters (such as Mean, Standard deviation, Correlation coefficient, covariance, and Entropy) that are commonly used to measure image quality, the values shown in Tables 1 and 2 were tabulated to show that the proposed method yielded optimal results with respect to all variables.

Table 1: Statistical parameters Analysis for spatial domain (simple image) fusion methods

\begin{tabular}{|c|l|l|l|l|l|}
\hline Parameter & Entropy & Mean & $\begin{array}{l}\text { Standard } \\
\text { Deviation }\end{array}$ & covariance & $\begin{array}{l}\text { Correlation } \\
\text { coefficient }\end{array}$ \\
\hline Minimum & 2.2768 & 8.7265 & 24.1416 & 1.7634 & 0.2949 \\
\hline Maximum & 3.1584 & 20.5613 & 30.1462 & 2.2983 & 0.2442 \\
\hline Average & 5.9203 & 24.6725 & 34.7358 & 3.2628 & 0.0540 \\
\hline PCA & 6.7503 & 60.3849 & 20.4371 & 6.7894 & 0.2944 \\
\hline
\end{tabular}


Table 2: Statistical parameters Analysis for Transform domain fusion methods

\begin{tabular}{|c|c|c|c|c|c|}
\hline Parameter & Entropy & Mean & $\begin{array}{c}\text { Standard } \\
\text { Deviation }\end{array}$ & covariance & $\begin{array}{c}\text { Correlation } \\
\text { coefficient }\end{array}$ \\
\hline Discrete wavelet & 6.1519 & 44.2813 & 58.1302 & 7.1171 & 0.3456 \\
\hline $\begin{array}{c}\text { Laplacian } \\
\text { Pyramid }\end{array}$ & 6.0793 & 25.4532 & 58.2579 & 2.0563 & 0.5546 \\
\hline $\begin{array}{c}\text { Gradient } \\
\text { pyramid }\end{array}$ & 5.9196 & 19.2255 & 20.5991 & 3.3265 & 0.4079 \\
\hline $\begin{array}{c}\text { Orthogonal } \\
\text { Bi orthogonal }\end{array}$ & 6.0039 & 32.8347 & 30.2884 & 2.0293 & 0.0526 \\
\hline Trous & 6.0042 & 32.8347 & 30.2884 & 2.0293 & 0.5675 \\
\hline Haar & 6.0043 & 32.8347 & 30.2884 & 2.0293 & 0.5675 \\
\hline $\begin{array}{c}\text { Daubechies } \\
\text { Coiflets }\end{array}$ & 6.9411 & 65.5645 & 57.9220 & 7.2243 & 0.2890 \\
\hline $\begin{array}{c}\text { Womplex } \\
\text { Mavelet }\end{array}$ & 6.9305 & 68.1157 & 64.6418 & 7.9678 & 0.2072 \\
\hline $\begin{array}{c}\text { Wavelet } \\
\text { UWPC }\end{array}$ & 6.9400 & 75.2844 & 64.6418 & 7.9643 & 0.3110 \\
\hline $\begin{array}{c}\text { Symlet } \\
\text { Dual Tree }\end{array}$ & 6.9888 & 65.6741 & 60.1758 & 6.7909 & 0.3192 \\
\hline
\end{tabular}

\section{Conclusion}

Initially, work is done to build an alternate and efficient fusion method in comparison to the existing fusion methods presented in the literature review and in the first portion. In this section, a novel methodology is introduced using a block diagram to achieve better outcomes than existing methods. The final section of the work deals with the experimental analysis on various wavelet transform methods and applying canny edge detection on resultant fused images and analyzing the output visually as well as statistically with the help of quality measuring parameters (such as Mean, Standard deviation, Correlation coefficient, covariance, Entropy) that are commonly used to measure image quality, tabulate the values. In the future, the combination of revolutionary approaches involving 3-D MRI and CT may result in a valuable tool for the real-time processing of fusion of MRI and CT, which will aid clinicians in the diagnosis of an emergency patient. 


\section{References}

[1.] A.C. Riegel, A.M. Berson, S. Destian, T. Ng, L.B. Tena, R.J. Mitnick, et al., "Variability of Gross Tumor Volume Delineation in Head-and-Neck Cancer using CT and PET/CT fusion", International Journal of Radiation Oncology Biology Physics, vol. 65, no. 3, pp. 726-732, 2006

[2.] A. Deng, Jin Wu and S. Yang, "An Image Fusion Algorithm Based on Discrete Wavelet Transform and Canny Operator", Advance Research on Computer Education Simulation and Modelling Communication in Computer and Information Science, vol. 175, pp. 32-38, 2011

[3.] Chaunte W. Lacewell, Mohamed Gebril, Ruben Buaba and Abdollah Homaifar, "Optimization of Image Fusion Using Genetic Algorithms and Discrete Wavelet Transform”, IEEE 2010, pp. 116121

[4.] Chu-Hui Lee and Zheng-Wei Zhou, "Comparison of Image Fusion based on DCT-STD and DWTSTD", International Multi-Conference of Engineers and Computer scientists 2012 Vol. I, IMECS2012, Hong Kong, March 14-16, 2012

[5.] K. Kannan, S. A. Perumal and K. Arulmozhi, "Performance Comparison of Various Levels of Fusion of Multi-focused Images using Wavelet Transform", International Journal of Computer Applications, vol. 1, no. 6, pp. 71-78, 2010

[6.] Liu Y., Cheng H., Huang J., Zhang Y., Tang X., and Tian J., "An Effective Non-rigid Registration Approach for Ultrasound Image Based on „Demons "e Algorithm,” Journal of Digital Imaging, vol. 26, no. 3, pp. 521-529, 2013

[7.] Maintz J. and Viergever M., "A Survey of Medical Image Registration,” Medical Image Analysis, vol. 2, no. 1, pp. 1-36, 1998

[8.] Marcio H. Ribeiro Sales, Carlos M. Souza, Jr., and Phaedon C. Kyriakidis, "Fusion of MODIS Images Using Kriging With External Drift", IEEE Transactions On Geoscience And Remote Sensing, VOL. 51, NO. 4, APRIL 2013, pp. 2250-2259

[9.] Naidu V. and Raol J., "Pixel-Level Image Fusion Using Wavelets and Principal Component Nalysis,” Defence Science Journal, vol. 58, no. 3, pp. 338-352, 2008

[10.] Parmar, Kiran, Rahul K. Kher, and Falgun N. Thakkar. "Analysis of CT and MRI Image Fusion Using Wavelet Transform." IEEE International Conference on Communication Systems and Network Technologies (CSNT), pp. 124-127, 11-13 May, 2012

[11.] R. Singh and A. Khare, "Fusion of Multimodal Images using Daubechies Complex Wavelet Transform-A Multiresolution Approach", Elsevier-Information Fusion, vol. 19, pp. 4960, 2014

[12.] Rajeswara Mahidar P, N.V.G.Prasad, V.L.N sastry , "A Novel Noisy Image Fusion Technique Using Dual-Tree Complex Wavelets Transform”; IJEST, Vol. 3 No. 6, June 2011

[13.] S. K. Sadhasivam, M. K. Keerthivasan and S Muttan, "Implementation of Max Principle with PCA in Image Fusion for Surveillance and Navigation Application", Electronic Letters on Computer Vision and Image Analysis, vol. 10, no. 1, pp. 1-10, 2011 